\title{
Spin signatures of exchange-coupled triplet pairs formed by singlet fission
}

\author{
Sam L. Bayliss, ${ }^{1}$ Leah R. Weiss, ${ }^{1}$ Akshay Rao, ${ }^{1}$ Richard H. \\ Friend, ${ }^{1}$ Alexei D. Chepelianskii, ${ }^{2}$ and Neil C. Greenham ${ }^{1, *}$ \\ ${ }^{1}$ Cavendish Laboratory, University of Cambridge, Cambridge, CB3 OHE, U.K \\ ${ }^{2}$ LPS, Univ. Paris-Sud, CNRS, UMR 8502, F-91405, Orsay, France
}

\begin{abstract}
We study the effect of an exchange interaction on the magnetic field dependent photoluminescence in singlet fission materials. We show that for strongly interacting triplet exciton pairs (intertriplet exchange interaction greater than the intratriplet spin-dipolar interaction), quantum beating and magnetic field effects vanish apart from at specific magnetic fields where singlet and quintet levels are mixed by a level anticrossing. We characterize these effects and show that the absence of a magnetic field effect or zero-field quantum beats does not necessarily mean that fission is inoperative. These results call for a reconsideration of the observations that are considered hallmarks of singlet fission, and demonstrates how the spin coherence and exchange coupling of interacting triplet pairs can be measured through magneto-photoluminescence experiments.
\end{abstract}

PACS numbers: 81.05.Fb, 88.40.jr, 71.35.-y, 78.20.Ls

\section{INTRODUCTION}

Magnetic field effects have been instrumental in understanding a range of phenomena in organic materials, ${ }^{1}$ from charge transport and recombination in organic semiconductors, ${ }^{2-6}$ to mechanisms of avian navigation. ${ }^{7}$ Of pivotal importance to the field of organic solar cells were pioneering early experiments on the changes in emission induced by a magnetic field in crystalline tetracene. $^{8-10}$ These experiments provided direct evidence for singlet fission - the splitting of one singlet exciton into two triplet excitons ${ }^{11,12}$ - and lay the foundation for the resurgent interest in this process as a means to boost the efficiency of solar energy harvesting. ${ }^{13-15}$ Following these early developments, magnetic field effects have been a key tool in identifying and understanding singlet fission, both in disordered and crystalline materials ${ }^{16,17}$ and in photovoltaic devices. ${ }^{18,19}$

In both early and recent work, the prevailing assumption has been that triplet pairs interact only weakly more specifically, that the spin-dipolar interaction between electron and hole within a single triplet exciton (the zero-field splitting) dominates any spin-dependent interaction between the triplets, such as an exchange coupling. Under these conditions, magnetic field effects on photoluminescence or photocurrent will display a characteristic saturation when the Zeeman interaction from an external field is larger than the zero-field splitting interaction, ${ }^{20}$ and observation of coherent oscillations in time-resolved photoluminescence experiments (quantum beating) is possible at zero magnetic field. ${ }^{21}$

Motivated by the central role that triplet pairs play in utilizing fission, here we study the strongly interacting regime where intertriplet exchange coupling dominates the intratriplet dipolar interaction (which is typically $\sim 5 \mu \mathrm{eV}$ for organic triplet excitons $\left.{ }^{22-26}\right)$. We demonstrate the consequences of this for identifying and studying singlet fission, and show that this strongly interacting regime gives rise to a very different set of spin signatures than those usually assumed. Firstly we discuss steady-state magnetic field effects, which we note have also recently been independently invoked to explain magneto-photoluminescence experiments in 1,6diphenyl-1,3,5-hexatriene, ${ }^{27}$ before providing a detailed description of time-domain quantum beating: an unambiguous probe of strongly coupled triplet pairs. These effects provide a way of determining the exchange coupling and spin coherence time for interacting triplet pairs, and challenge the conventional use of low magnetic field effects as hallmarks of singlet fission. While we study effects in singlet fission materials, our results are also applicable to triplet-triplet annihilation in light-emitting diodes as well as in upconversion photovoltaics. ${ }^{28-31}$

\section{STEADY-STATE MAGNETIC FIELD EFFECTS}

We start by considering the effect of a static magnetic field on the photoluminescence (PL) of a singlet fission material. The kinetic scheme [sketched in Fig. 1(a)], follows the treatment outlined by Merrifield et al.:20

$$
\begin{array}{rll}
G & \searrow & \gamma_{+} \alpha_{i} \\
& S & \underset{\rightleftharpoons}{\rightleftharpoons} \\
\gamma_{r} & \downarrow & \gamma_{-} \alpha_{i}
\end{array}\left\{P_{i}\right\} \stackrel{\gamma_{d}}{\rightarrow} T+T .
$$

A singlet exciton $S$ is generated at rate $G$ and can radiatively decay at rate $\gamma_{r}$, or undergo fission to form one of the triplet-pair eigenstates $\left|P_{i}\right\rangle$ at rate $\gamma_{+} \alpha_{i}$, where $\alpha_{i}$ is the overlap between the singlet $|S\rangle$ and triplet-pair spin wavefunctions: $\alpha_{i}=\left|\left\langle S \mid P_{i}\right\rangle\right|^{2}$. The triplet pair can either reform the singlet exciton at rate $\gamma_{-} \alpha_{i}$, or dissociate into free triplets at rate $\gamma_{d}$.

The triplet-pair states $\left\{\left|P_{i}\right\rangle\right\}$ and hence their singlet projections $\left\{\alpha_{i}\right\}$ are determined by the pair Hamiltonian

$$
\hat{H}=\underbrace{J \hat{\mathbf{S}}_{a} \cdot \hat{\mathbf{S}}_{b}}_{\hat{H}_{e x}}+\sum_{i=a, b} \underbrace{g \mu_{B} \mathbf{B} \cdot \hat{\mathbf{S}}_{i}}_{\hat{H}_{i, B}}+\underbrace{D\left(\hat{S}_{i, z}^{2}-\hat{\mathbf{S}}_{i}^{2} / 3\right)}_{\hat{H}_{i, \mathrm{zfs}}},
$$



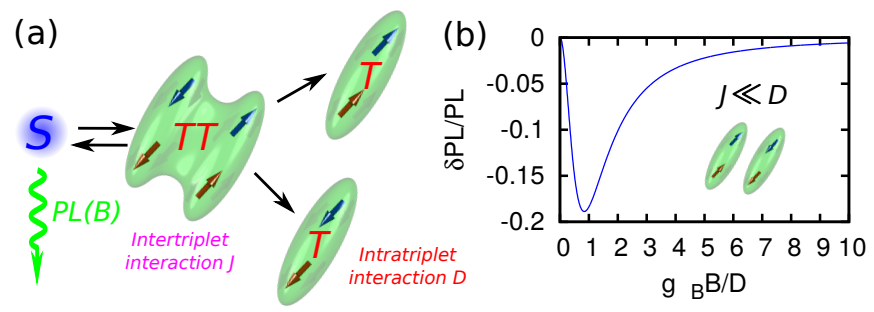

FIG. 1. (Color online.) (a) Schematic of the kinetic scheme used to calculate the magnetic field effects on the photoluminescence $(\mathrm{PL})$. The triplets are subject to an intertriplet exchange interaction $J$ and an intratriplet spin dipolar interaction, characterized by the zero-field splitting parameter $D$, as well as the Zeeman interaction due to an applied magnetic field $B$. (b) Magnetic field effect for weakly coupled $(J \ll D)$ triplet pairs, where $\delta \mathrm{PL} / \mathrm{PL}=(\mathrm{PL}(B)-\mathrm{PL}(0)) / \mathrm{PL}(0)$. The effect saturates on a field scale set by the zero-field splitting $D$ which is typically $\sim 50 \mathrm{mT}$.

where $\hat{H}_{e x}$ is the exchange interaction with coupling parameter $J, \hat{\mathbf{S}}_{i}=\left(\hat{S}_{i, x}, \hat{S}_{i, y}, \hat{S}_{i, z}\right)$ are the spin operators for the two triplets $(i=a, b), \hat{H}_{i, \mathrm{zfs}}$ is the intratriplet zero-field splitting interaction, ${ }^{32}$ with zero-field splitting parameter $D$, and $\hat{H}_{i, B}$ is the Zeeman interaction from an external magnetic field $\mathbf{B}$, where $g$ is the Landé $g$-factor and $\mu_{B}$ the Bohr magneton.

Solving the kinetic scheme (Eq. 1) using the wavefunctions $\left\{\left|P_{i}\right\rangle\right\}$ determined by the pair Hamiltonian $\hat{H}$ gives the steady-state photoluminescence from the singlet exciton $\mathrm{PL}=\gamma_{r} S$. Assuming singlet fission is efficient so that it dominates radiative decay, i.e. $\gamma_{+} \gg \gamma_{r}$, we arrive at

$$
\mathrm{PL}=a\left(\sum_{i} \alpha_{i}\left(1+\epsilon \alpha_{i}\right)^{-1}\right)^{-1}
$$

where $a=\gamma_{r} G / \gamma_{+}$and $\epsilon=\gamma_{-} / \gamma_{d}$. Since the sum depends non-linearly on $\alpha_{i}$, changes in the triplet-pair eigenstates due to competition between different terms in the Hamiltonian lead to changes in the singlet projections $\left\{\alpha_{i}\right\}$, and hence a magnetic field effect.

Fig. 1(b) reviews the conventionally assumed scenario of weak intertriplet coupling $(J \ll D)$ showing the normalized changes in photoluminescence $\delta \mathrm{PL} / \mathrm{PL}=(\mathrm{PL}(B)$ $\mathrm{PL}(0)) / \mathrm{PL}(0)$ as a function of magnetic field. (Unless otherwise stated, the angle between the triplet zero-field splitting tensor and the magnetic field $\theta=\pi / 4$ and $\epsilon=1$. Note that $\epsilon$ determines the magnitude of the magnetic field effect, but does not influence the lineshape.) The emission shows a characteristic reduction and recovery with a characteristic field scale of $\sim D / g \mu_{B}$, which is typically $\sim 50 \mathrm{mT}$ for triplet excitons in organic semiconductors (i.e. $D \sim 5 \mu \mathrm{eV}) .{ }^{22-26}$ As described previously, ${ }^{20}$ this behavior results from the competition between the zerofield splitting and Zeeman interactions which changes the spin projections $\left\{\alpha_{i}\right\}$, and hence the population of the emissive singlet state. ${ }^{33}$
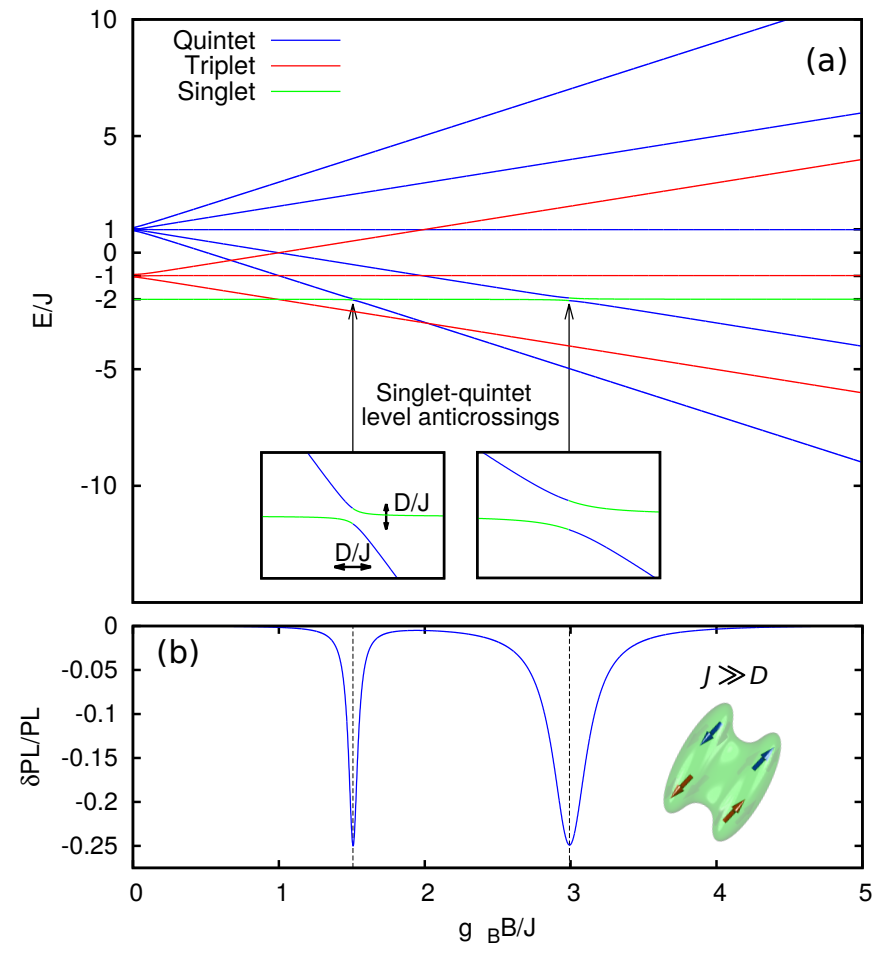

FIG. 2. (Color online.) Magnetic field effects of exchangecoupled triplet pairs formed by singlet fission. Energy level diagram (a) and magnetic field effect (b), for strongly coupled $(J / D=10)$ triplet pairs. The singlet, triplet and quintet manifolds are separated by the exchange interaction. A magnetic field effect is only observed in the region of the singletquintet level anticrossings [insets of (a)], where the Zeeman interaction cancels the effect of the exchange interaction.

Fig. 2(b) shows the strongly exchange-coupled limit $J \gg D$, which exhibits a very different behavior - a magnetic field effect only occurs at the specific fields which correspond to level anticrossings between the quintet and singlet manifolds [Fig. 2(a)]. At zero magnetic field, a strong exchange coupling means that all nine triplet-pair states have a well-defined spin multiplicity, forming singlet $(S=0)$, triplet $(S=1)$ and quintet $(S=2)$ manifolds (here $S$ denotes the total spin). In other words, spin and energy eigenstates coincide. At finite magnetic fields, and away from the level anticrossings, the states retain their multiplicity since total spin remains a good quantum number. There is no change in the singlet projections $\left\{\alpha_{i}\right\}$, and hence no change in emission. However, when the Zeeman interaction compensates the exchange interaction, quintet states are brought into degeneracy with the singlet state. The zero-field splitting interaction mixes these states to form singlet-quintet mixtures $\left|\psi_{m}^{( \pm)}\right\rangle=\frac{1}{\sqrt{2}}\left(\left|Q_{m}\right\rangle \pm|S\rangle\right)$ where $\left|Q_{m}\right\rangle$ is the quintet state with spin projection $m=-2$ or -1 . These mixed states do not have a well-defined spin multiplicity $(\alpha=1 / 2$ for both states): the singlet projections $\left\{\alpha_{i}\right\}$ therefore change from their off-resonance values of $\alpha_{i}=\delta_{i S}$ (where 
$S$ denotes the singlet state), resulting in a dip in the emission.

These anticrossing resonances arise when the Zeeman energy matches the separation between singlet and quintet states at zero field. This separation has a contribution of $3 J$ from the exchange interaction plus a contribution from the zero-field splitting. In the strongly coupled limit $(J \gg D)$ considered here, the zero-field splitting $\hat{H}_{\mathrm{zfs}}=\sum_{i=a, b} \hat{H}_{i, \mathrm{zfs}}$ can be treated as a perturbation on the exchange interaction $\hat{H}_{e x}$, which gives an additional energy offset due to the zero-field splitting of $\left\langle Q_{m}\left|\hat{H}_{\mathrm{zfs}}\right| Q_{m}\right\rangle$ for state $\left|Q_{m}\right\rangle$. Taking this into account, the magnetic field positions of the level anticrossings $B_{m}$ are determined by $m g \mu B_{m}=3 J+\left\langle Q_{m}\left|\hat{H}_{\mathrm{zfs}}\right| Q_{m}\right\rangle$. With an angle $\theta$ between the principal axis of the zero-field splitting tensor and $\mathbf{B}$, we arrive at

$$
\begin{aligned}
& B_{-2}=\frac{3 J}{2}+\frac{D}{12}(1+3 \cos (2 \theta)) \simeq \frac{3 J}{2} \\
& B_{-1}=3 J-\frac{D}{12}(1+3 \cos (2 \theta)) \simeq 3 J .
\end{aligned}
$$

The exchange coupling $J$ can therefore be directly determined from the resonance positions.

The magnitude of the effects shown in Fig. 2(b) can be rationalized as follows. At zero field the singlet projections are $\alpha_{i}=\delta_{i S}$ and hence $\operatorname{PL}(0)=a(1+\epsilon)$. At the respective level anticrossings, we have $\alpha=1 / 2$ for the states $\left|\psi_{m}^{( \pm)}\right\rangle$which gives $\operatorname{PL}\left(B_{m}\right)=a(1+\epsilon / 2)$ and hence

$$
\frac{\delta \mathrm{PL}\left(B_{m}\right)}{\mathrm{PL}}=\frac{\epsilon}{2(1+\epsilon)} .
$$

With $\epsilon=1, \delta \mathrm{PL}\left(B_{m}\right) / \mathrm{PL}=-1 / 4$, as seen in Fig. 2(b).

The widths of the PL resonances are determined by the zero-field splitting $D$ since the mixing between singlet and quintet states is only effective when the separation between these levels $\left|E\left(Q_{i}\right)-E(S)\right| \sim D$ [Fig. 2(a), inset], where $E(\psi)=\langle\psi|\hat{H}| \psi\rangle$. The width of the lower-field resonance at $g \mu_{B} B \simeq 3 J / 2$ is twice as narrow as the resonance at $g \mu_{B} B \simeq 3 J$ since the energy of the $\left|Q_{-2}\right\rangle$ state shifts twice as fast with magnetic field compared to the $\left|Q_{-1}\right\rangle$ state, and hence becomes detuned from resonance twice as fast as $B$ is increased.

Fig. 3(a) shows the dependence of the high field resonances on $\theta$, the angle between the magnetic field and the principal axis of the zero-field splitting tensor. The two resonances show distinct behaviors as a result of the different way that $\hat{H}_{\text {zfs }}$ couples the two quintet states $\left|Q_{-2}\right\rangle,\left|Q_{-1}\right\rangle$ to the singlet state $|S\rangle$

$$
\begin{aligned}
& \left\langle Q_{-2}\left|\hat{H}_{\mathrm{zfs}}\right| S\right\rangle=\frac{D}{\sqrt{3}} \sin ^{2}(\theta) \\
& \left\langle Q_{-1}\left|\hat{H}_{\mathrm{zfs}}\right| S\right\rangle=\frac{D}{\sqrt{3}} \sin (2 \theta) .
\end{aligned}
$$

This angular-dependent coupling determines the degree of hybridization between singlet and quintet states which
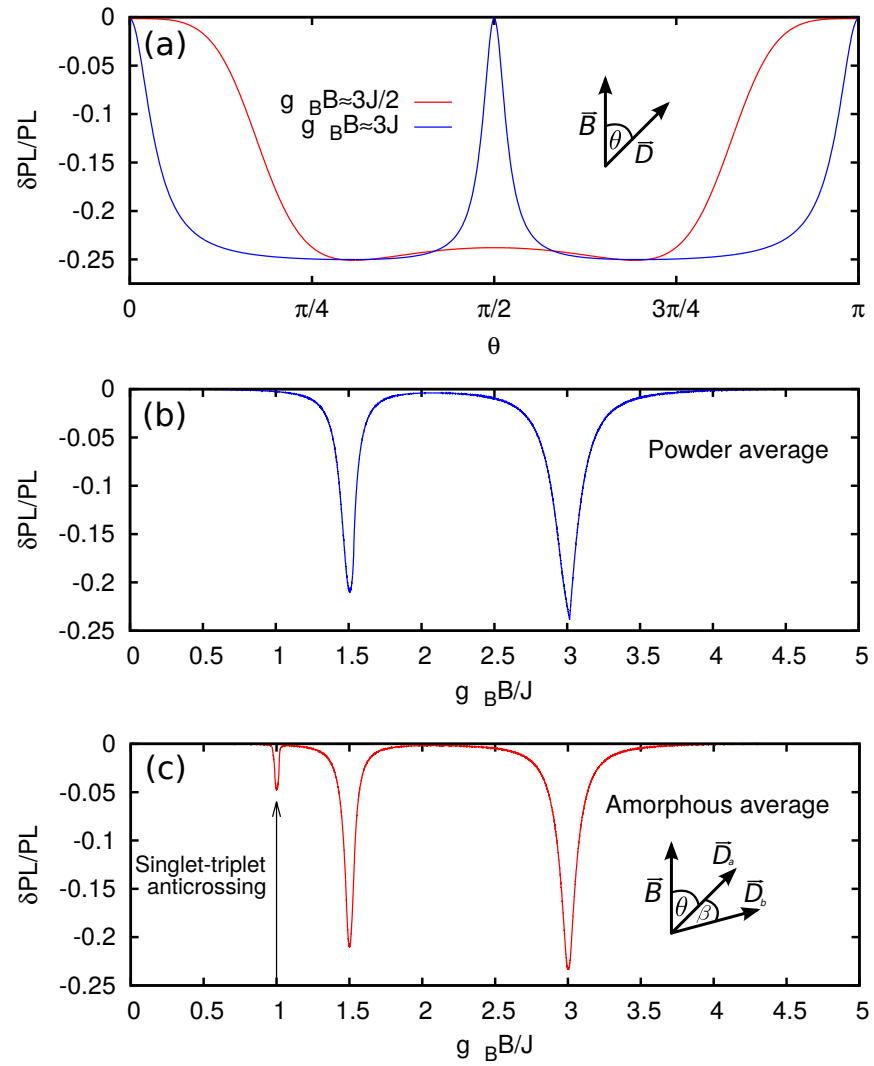

FIG. 3. (Color online.) (a) Angular dependence of the highfield resonances: magnetic field effect shown as a function of the angle $\theta$ between the magnetic field $\mathbf{B}$ and the zero-field splitting principal axis $\vec{D}$. (b) Powder average of high-field resonances. Magnetic field effect calculated by averaging the response of an ensemble of randomly oriented triplet pairs. (c) Effect of inequivalent triplets on the magnetic field effect. By including an angle $\beta$ between the two triplets, the particle-exchange symmetry is broken which allows an additional singlet-triplet level anticrossing to be observed at $g \mu_{B} B \simeq J$. Simulations are shown for an amorphous average in which the two triplets are randomly oriented with respect to each other and the magnetic field.

determines the singlet projections $\left\{\alpha_{i}\right\}$. These ultimately determine the PL through Eq. 3, giving rise to the angular dependence in Fig. 3(a). For the resonance at $g \mu_{B} B \simeq 3 J / 2$ the coupling between $\left|Q_{-2}\right\rangle$ and $|S\rangle$, and hence any magnetic field effect, vanishes for $\theta=0, \pi$, while for the resonance at $g \mu_{B} B \simeq 3 J$, the $\sin (2 \theta)$ dependence of the coupling between $\left|Q_{-1}\right\rangle$ and $|S\rangle$ means that the effect also vanishes for $\theta=\pi / 2$.

The average of the magneto-PL over all orientations (powder average) is shown in Fig. 3(b). This represents what would be measured in a sample with randomly oriented triplet pairs, and shows that the essential features of the crystalline case shown in Fig. 2(b) are preserved. The powder average has slightly reduced magnitudes of $\delta \mathrm{PL} / \mathrm{PL}$ compared to the crystalline case due to the fact that the singlet-quintet mixing induced 
by $\hat{H}_{\mathrm{zfs}}$ is not effective for all angles [Fig. 3(a)]. The weaker angular dependence of the higher-field resonance at $g \mu_{B} B \simeq 3 J$ compared to the lower-field resonance at $g \mu_{B} B \simeq 3 J / 2$ leads to the slight asymmetry between the dips and means that $\delta \mathrm{PL} / \mathrm{PL}$ for the higher-field anticrossing is closer to $-1 / 4$ than for the lower-field one.

When the two triplets in a pair are equivalent, i.e. when their zero-field splitting tensors have the same orientation and $D$ values, and their exciton $g$-values are identical, the total Hamiltonian $\hat{H}$ is symmetric under interchange of triplet excitons. This means that the pair spin eigenstates are either symmetric (singlet and quintet manifolds) or antisymmetric (triplet manifold) under particle exchange. ${ }^{20,34}$ The singlet-triplet level crossing at $g \mu_{B} B / J \simeq 1$ shown in Fig. 2(a) therefore does not lead to a resonance - despite the degeneracy between $\left|T_{-1}\right\rangle$ and $|S\rangle, \hat{H}_{\mathrm{zfs}}$ cannot mix states with different symmetry. However, when triplets are inequivalent, this mixing becomes possible and this anticrossing can be observed. Fig. 3(c) shows the magneto-PL for triplets with the same $D$ values but which are randomly oriented with respect to each other and the external field, i.e. a completely amorphous average. By breaking the particle exchange symmetry, singlet and triplet levels can be mixed, leading to an additional avoided crossing at $g \mu_{B} B / J \simeq 1$ and hence a resonant dip in the PL.

Note that morphologies consisting of rotationally inequivalent molecules (e.g. the herringbone structure found in crystalline tetracene) do not necessarily imply that the triplets are inequivalent. Provided the hopping rate between inequivalent sites is sufficiently fast i.e. $\tau_{h o p}^{-1} \gg D_{m o l} / \hbar$, where $\tau_{h o p}$ is the hopping time and $D_{m o l}$ is the molecular zero-field splitting, each triplet will experience an average over these sites, and therefore be magnetically equivalent. ${ }^{35}$ In addition, electronic coupling will be sensitive to the relative orientation of neighboring molecules, imposing constraints on the range of angles where singlet fission and triplet fusion are effective and thus filtering which molecular orientations contribute to the magneto-PL. ${ }^{17,36}$ The singlet-triplet anticrossing resonance in Fig. 3(c) provides a way of identifying to what extent fission and fusion are occurring between inequivalent triplets.

Conformational disorder could also lead to a distribution of exchange-coupling parameters. The anticrossing resonances would then become a weighted sum of the contributions from pairs with different $J$-values i.e. $\delta P L \rightarrow \int \delta P L(J) g(J) d J$ where $g(J)$ is the distribution of $J$-values. The extent to which this will modify magnetic field effects will depend on both the level of disorder and also, as discussed above, how site-selective fission and fusion are.

We emphasize that the strongly interacting regime outlined here occurs when the exchange interaction is larger than the zero-field splitting, which is typically $\sim 5 \mu \mathrm{eV}$ for organic triplet excitons. ${ }^{22-26}$ This regime can therefore be reached for an intertriplet exchange interaction which is low compared to the $\sim \mathrm{eV}$ energy scale typical for the singlet-triplet exchange interaction within a single exciton. ${ }^{37,38}$ In our recent electron spin resonance experiments on a tetracene derivative, ${ }^{39}$ we found evidence for exchange-coupled triplet pairs and estimated an upper bound of $J / g \mu_{B} \lesssim 36 \mathrm{~T}$ (i.e. $J \lesssim 4 \mathrm{meV}$ ), suggesting the high-field resonances outlined here might be amenable to experimental measurement. We also note that analogous hyperfine-mediated singlet-triplet level anticrossings have proven to be a useful tool to estimate the exchange coupling in radical ion pairs. ${ }^{40}$

While we have studied the magneto-PL for a neat singlet fission material, the results outlined here also apply to the photocurrent in singlet fission solar cells, and nanocrystal emission in triplet-transfer systems, where magnetic field effects are often used as a test of whether singlet fission is operative. ${ }^{14,15,18}$ In addition, these results are applicable to the delayed fluorescence from triplet-triplet annihilation in upconversion systems and light-emitting diodes. ${ }^{28-31}$

\section{QUANTUM BEATS}

We now consider the effect of a strong exchange coupling $(J \gg D)$ on the time-domain photoluminescence, in analogy to the weakly coupled scenario. ${ }^{21,41,42}$ We find the time-dependence of the triplet-pair density matrix $\hat{\rho}(t)$ as a function of magnetic field by solving the following equation of motion

$$
\partial_{t} \rho_{i j}(t)=-i \omega_{i j} \rho_{i j}(t)-\frac{\gamma_{S}}{2}\left\{\hat{P}_{S}, \hat{\rho}(t)\right\}_{i j}
$$

Here we work in the basis in which the total Hamiltonian is diagonal with eigenvalues $\left\{\hbar \omega_{i}\right\}, \omega_{i j}=\omega_{i}-\omega_{j}$, and $\gamma_{S}$ is the recombination rate via the singlet channel, ${ }^{43}$ with $\hat{P}_{S}=|S\rangle\langle S|$ the singlet projector. The curly braces denote the anticommutator.

We solve Eq. 9 with triplet pairs initialized in a singlet state by fission i.e. $\hat{\rho}(0)=\hat{P}_{S}$, and determine the singlet content of the triplet pair as a function of time $\left\langle\hat{P}_{S}\right\rangle=\operatorname{Tr}\left(\hat{\rho}(t) \hat{P}_{S}\right)$, where $\operatorname{Tr}$ denotes the trace. Since the singlet content of a triplet pair determines their ability to recombine emissively, $\left\langle\hat{P}_{S}\right\rangle$ will therefore determine the photoluminescence. (For clarity, we assume that $\gamma_{S}$ is faster than spin decoherence and dissociation rates, which would feature as additional damping terms.)

Fig. 4(a) shows $\left\langle\hat{P}_{S}\right\rangle$ as a function of time and magnetic field for $J / D=10$ and $\gamma_{S}=\omega_{D} / 10$, where $\omega_{D}=D / \hbar$. Coherent oscillations only occur at the level anticrossings shown in Fig. 2(a) and quickly die away from these positions. This differs markedly from the weakly coupled $(J \ll D)$ case where quantum beats occur at arbitrary magnetic fields. ${ }^{16,21,42}$

The essential behavior observed in Fig. 4(a) arises as follows. As described in II, away from the level anticrossings a strong exchange coupling locks triplet pairs in an overall spin-singlet state. The singlet content of a triplet 
(a)

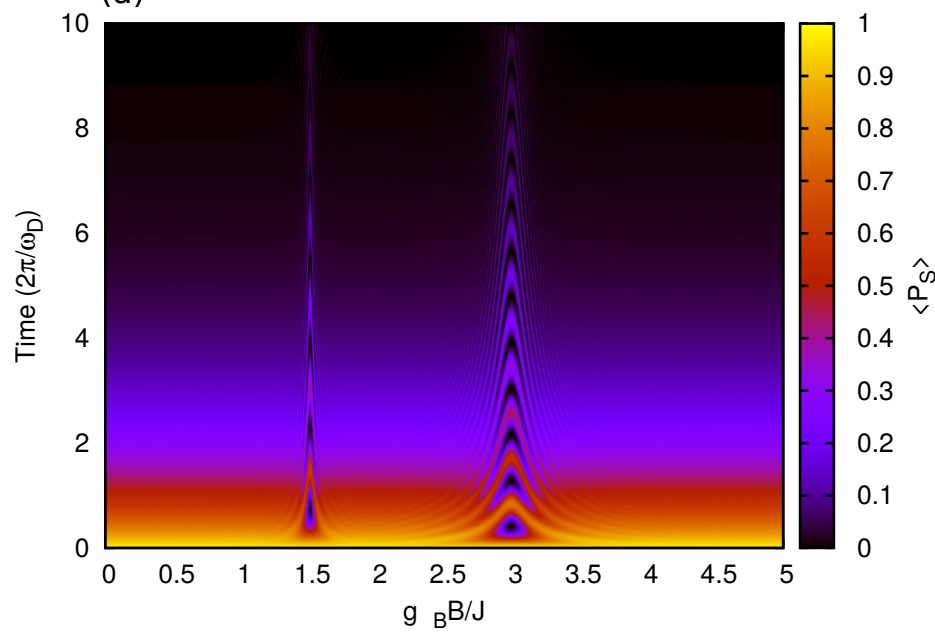

(b)
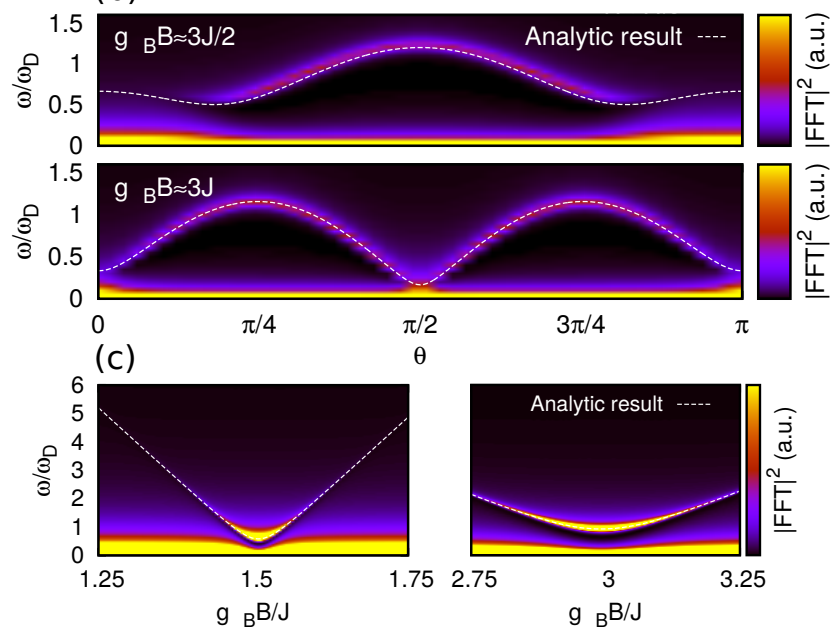

FIG. 4. (Color online.) Quantum beats of exchange-coupled triplet pairs. (a) Singlet content of the triplet pair $\left\langle\hat{P}_{S}\right\rangle$ as a function of time and magnetic field following fission. Away from the singlet-quintet level anticrossings, the triplets remain in the pure spin-singlet state following fission and no beats are observed. Near the level anticrossings, the pure singlet state formed by singlet fission is no longer an energy eigenstate, and the singlet character of the pair oscillates in time. The oscillation frequency is characterized by the zero-field splitting $\omega_{D}=D / \hbar$. [Simulations shown for a single triplet-pair orientation, as in Fig. 2(b).] (b)/(c) Fast-Fourier transform (FFT) of the singlet-character oscillations giving the beat frequencies $\omega$ for the two high-field resonances. In (b) the beat frequencies are shown as a function of the orientation of the triplet pair with respect to the magnetic field. In (c) the beat frequencies are shown as a function of magnetic field for a fixed orientation $\theta=\pi / 4$. Analytic results for the beat frequencies are overlaid in dashed lines.

pair $\left\langle\hat{P}_{S}\right\rangle$ does not oscillate in time, and instead monotonically decreases as pairs recombine. At the level anticrossings however, the hybrid states $\left|\psi_{m}^{( \pm)}\right\rangle=\frac{1}{\sqrt{2}}\left(\left|Q_{m}\right\rangle \pm|S\rangle\right)$ are formed, separated in energy by the zero-field splitting:

$$
\begin{aligned}
\left\langle\psi_{-2}^{(+)}\left|\hat{H}_{\mathrm{zfs}}\right| \psi_{-2}^{(+)}\right\rangle-\left\langle\psi_{-2}^{(-)}\left|\hat{H}_{\mathrm{zfs}}\right| \psi_{-2}^{(-)}\right\rangle & =\frac{2 D}{\sqrt{3}} \sin ^{2}(\theta) \equiv \hbar \omega_{-2} \\
\left\langle\psi_{-1}^{(+)}\left|\hat{H}_{\mathrm{zfs}}\right| \psi_{-1}^{(+)}\right\rangle-\left\langle\psi_{-1}^{(-)}\left|\hat{H}_{\mathrm{zfs}}\right| \psi_{-1}^{(-)}\right\rangle & =\frac{2 D}{\sqrt{3}} \sin (2 \theta) \equiv \hbar \omega_{-1} .
\end{aligned}
$$

Fission generates pairs in an overall singlet state $\mid \Psi(t=$ $0)\rangle=|S\rangle=\frac{1}{\sqrt{2}}\left(\left|\psi_{m}^{(+)}\right\rangle-\left|\psi_{m}^{(-)}\right\rangle\right)$, which evolves in time to generate a relative phase shift of $\omega_{m} t$ between $\left|\psi_{m}^{( \pm)}\right\rangle$ i.e. $|\Psi(t)\rangle \propto\left(\left|\psi_{m}^{(+)}\right\rangle-e^{+i \omega_{m} t}\left|\psi_{m}^{(-)}\right\rangle\right)$. The singlet projection therefore oscillates in time with a frequency $\omega_{m}$ : $|\langle\Psi(t) \mid S\rangle|^{2} \propto\left(1+\cos \left(\omega_{m} t\right)\right)$, and beats are observed. (Note that in Fig. 4(a) the time integral of $\left\langle\hat{P}_{S}\right\rangle$ is independent of magnetic field i.e. $\int_{0}^{\infty}\left\langle\hat{P}_{S}\right\rangle d t=\gamma_{S}^{-1}$. This arises from the fact that all triplet pairs recombine via the singlet channel [Eq. 9].)

The angular dependence of the beat frequencies at the two magnetic field positions corresponding to the resonances is illustrated in Fig. 4(b). This shows the Fast Fourier Transform (FFT) of $\left\langle\hat{P}_{S}\right\rangle$, calculated by solving Eq. 9, as a function of $\theta$ and frequency $\omega$, overlaid with the above analytic results for the beat frequencies. In analogy with Fig. 3(a), this highlights the distinct angular dependence of the two resonances arising from the selection rules invoked by $\hat{H}_{\text {zfs }}$.

Fig. 4(c) shows the dependence of the beat frequencies on the magnetic field for a fixed orientation $(\theta=\pi / 4)$. Only two states are involved at each level anticrossing, and so we can restrict our ourselves to the relevant $2 \times 2$ subspaces $\left(\left|Q_{m}\right\rangle,|S\rangle\right)$. At the respective anticrossings, the reduced Hamiltonian becomes

$$
\hat{H}=\left(\begin{array}{cc}
E\left(Q_{m}\right) & V_{m} / 2 \\
V_{m} / 2 & E(S)
\end{array}\right)
$$

where $V_{m} / 2=\left\langle Q_{m}\left|\hat{H}_{\mathrm{zfs}}\right| S\right\rangle$, defined in Eqs. 7-8. The beat frequencies $\omega_{m}$ are determined by the difference in eigenvalues of Eq. 10

$$
\begin{aligned}
\hbar \omega_{m} & =\sqrt{\delta E_{m}^{2}+V_{m}^{2}} \\
& =\sqrt{\left(m g \mu_{B}\left(B_{m}-B\right)\right)^{2}+V_{m}^{2}},
\end{aligned}
$$

where $\delta E_{m}=E\left(Q_{m}\right)-E(S)$ and the $B_{m}$ are defined in Eqs. 4-5. These analytic results are plotted in Fig. 4(c) along with the FFT of the numerical simulations. The numerics show that the visibility of the beats decreases as the magnetic field is detuned away from the resonances a result of the reduced mixing between singlet and quintet states due to their increasing energy separation. This can also be seen from the eigenvectors of Eq. 10 which are given by $\left|\psi_{m}^{(a)}\right\rangle=\cos \left(\frac{\phi_{m}}{2}\right)\left|Q_{m}\right\rangle+\sin \left(\frac{\phi_{m}}{2}\right)|S\rangle$ and $\left|\psi_{m}^{(b)}\right\rangle=\sin \left(\frac{\phi_{m}}{2}\right)\left|Q_{m}\right\rangle-\cos \left(\frac{\phi_{m}}{2}\right)|S\rangle$ where $\tan \left(\phi_{m}\right)=$ $V_{m} / \delta E_{m}$. Neglecting decay terms, an initially generated singlet $|S\rangle=\sin \left(\frac{\phi_{m}}{2}\right)\left|\psi_{m}^{(a)}\right\rangle-\cos \left(\frac{\phi_{m}}{2}\right)\left|\psi_{m}^{(b)}\right\rangle$ will therefore 


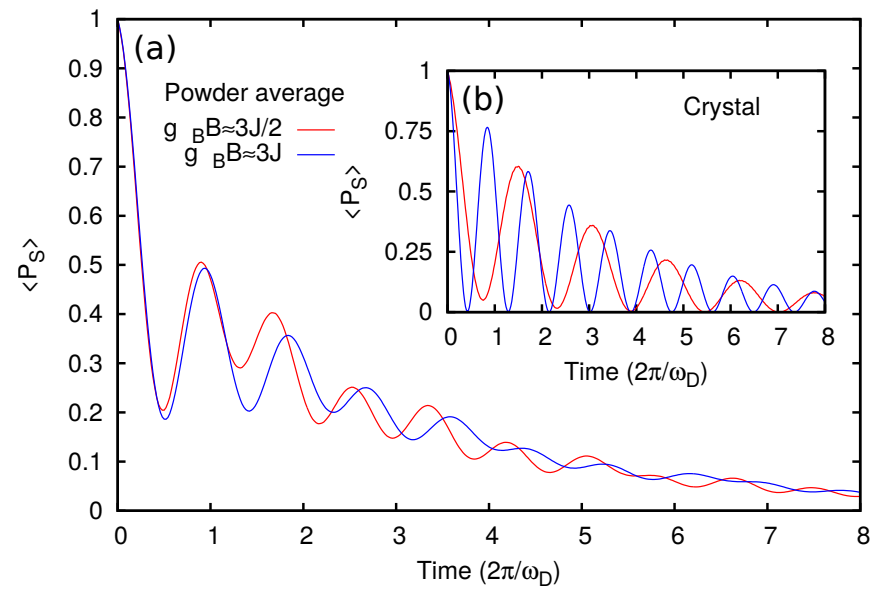

FIG. 5. (Color online.) (a) Powder average of quantum beats at the two high-field resonances. The oscillations are preserved after averaging over randomly oriented triplet pairs. The visibility of the oscillations diminishes more rapidly than in the crystalline case, shown in (b), but the beats are still visible. [(b) is calculated using the same parameters as (a) but with $\theta=\pi / 4]$.

give $\left\langle\hat{P}_{S}\right\rangle=\left(1+\frac{1}{2} \sin ^{2}\left(\phi_{m}\right)\left(\cos \left(\omega_{m} t\right)-1\right)\right)$ and hence a Fourier coefficient $\propto \sin ^{2}\left(\phi_{m}\right)=V_{m}^{2} /\left(V_{m}^{2}+\delta E_{m}^{2}\right)$. This expression highlights the dependence of the beating visibility on both the magnetic-field detuning $\left(\delta E_{m}\right)$ [Fig. 4(c)], and the matrix-element $V_{m}$, and hence $\theta$ dependence [Fig. 4(b)].

The beats are preserved after averaging over randomly oriented triplets [Fig. 5(a)]. The visibility of the oscillations is lost more rapidly than for the crystalline case with the same parameters [Fig. 5(b)] since triplets at different angles have different oscillation frequencies and therefore become out of phase with each other. However, oscillations are observable over several periods even after this ensemble averaging. Note that since $D$ sets the timescale of the beats, and also determines the effect of ensemble averaging on the dephasing, this preserved visibility is not dependent on the specific $D$ or $J$ parameters.

These high-field quantum beats provide a complementary set of experiments to the steady-state effects. Unlike the steady-state effects, which rely on a competition between dissociation and recombination, and hence vanish when triplets cannot separate, these time-domain experiments are sensitive to states which remain bound. (As described above, the time integral of Fig. 4(a) is fieldindependent.) We note that when the fission rate or excitation pulse is slow compared to $\omega_{D}=D / \hbar$, beats will not be observable in transient PL since triplet pairs will begin their oscillations out of phase. However, level- anticrossing magnetic field effects would still be visible in the incoherent PL dynamics, and would allow this bound regime to be revealed without having to observe beats. ${ }^{17}$

For simplicity, we have assumed that recombination is faster than spin decoherence. However, when spin decoherence dominates population kinetics, the decay of the beating will be determined by the triplet-pair spin coherence time. This allows magneto-PL to be used to extract the spin coherence time for strongly-coupled triplet pairs, as has been successfully demonstrated for the weakly coupled case. ${ }^{21,41,42}$ Furthermore, these beats provide an unambiguous way of verifying whether a steady-state magnetic field effect is due to a triplet-pair level anticrossing.

\section{CONCLUSION}

We have studied the magnetic field effects arising from the recombination of exchange-coupled triplet pairs formed by singlet fission. When the exchange interaction between triplets exceeds the intratriplet dipolar interaction, a key observation arises - the magnetic field effects and quantum beating which are often used as hallmarks of fission vanish at all fields apart from those which bring singlet and quintet (and possibly triplet) levels into neardegeneracy. This means that strongly-coupled fission systems display no quantum beating at zero magnetic field and can display no magnetic field effect in the typicallymeasured range of $B \sim 0-0.5 \mathrm{~T}$. The lack of a low $\left(B<J / g \mu_{B}\right)$ magnetic field effect, or zero-field quantum beats therefore does not necessarily imply that fission is inoperative and calls for a re-evaluation of what are considered the spin signatures of singlet fission. Our results demonstrate how the spin coherence and exchange coupling of interacting triplet pairs can be measured through magneto-photoluminescence, and could be particularly important for singlet fission dimers ${ }^{44-47}$ where excitonic confinement may lead to strong interactions. Finally, our recent estimate of $J / g \mu_{B} \lesssim 36 \mathrm{~T}$ in a singlet fission material ${ }^{39}$ suggests that the high-field resonances outlined here may be accessible experimentally, and that level anticrossing experiments could be important for studying molecular photovoltaic systems.

\section{ACKNOWLEDGMENTS}

We acknowledge support from the Engineering and Physical Sciences Research Council [Grants No. $\mathrm{EP} / \mathrm{G} 060738 / 1]$. There is no external research data as all necessary information is available in the paper.
* ncg11@cam.ac.uk

1 U. Steiner and T. Ulrich, Chem. Rev. 89, 51 (1989).
${ }^{2}$ T. D. Nguyen, G. Hukic-Markosian, F. Wang, L. Wojcik, X.-G. Li, E. Ehrenfreund, and Z. V. Vardeny, Nat. Mater. 
9, 345 (2010).

3 P. Janssen, M. Cox, S. Wouters, M. Kemerink, M. Wienk, and B. Koopmans, Nat. Commun. 4 (2013).

${ }^{4}$ P. A. Bobbert, T. D. Nguyen, F. W. A. van Oost, B. Koopmans, and M. Wohlgenannt, Phys. Rev. Lett. 99, 216801 (2007).

5 A. H. Devir-Wolfman, B. Khachatryan, B. R. Gautam, L. Tzabary, A. Keren, N. Tessler, Z. V. Vardeny, and E. Ehrenfreund, Nat. Commun. 5 (2014).

6 J. Wang, A. Chepelianskii, F. Gao, and N. C. Greenham, Nat. Commun. 3, 1191 (2012).

7 K. Maeda, K. B. Henbest, F. Cintolesi, I. Kuprov, C. T. Rodgers, P. A. Liddell, D. Gust, C. R. Timmel, and P. J. Hore, Nature 453, 387 (2008).

8 R. E. Merrifield, P. Akavian, and R. Groff, Chem. Phys. Lett. 3, 155 (1969).

9 N. Geacintov, M. Pope, and F. Vogel, Phys. Rev. Lett. 22, 593 (1969).

10 R. P. Groff, P. Avakian, and R. E. Merrifield, Phys. Rev. B 1, 815 (1970).

11 M. B. Smith and J. Michl, Chem. Rev. 110, 6891 (2010).

12 M. B. Smith and J. Michl, Annu. Rev. Phys. Chem. 64, 361 (2013).

13 P. J. Jadhav, A. Mohanty, J. Sussman, J. Lee, and M. A. Baldo, Nano Lett. 11, 1495 (2011).

14 N. J. Thompson, M. W. B. Wilson, D. N. Congreve, P. R. Brown, J. M. Scherer, T. S. Bischof, M. Wu, N. Geva, M. Welborn, T. Van Voorhis, V. Bulović, M. G. Bawendi, and M. A. Baldo, Nat. Mater. 13, 1039 (2014).

15 M. Tabachnyk, B. Ehrler, S. Gélinas, M. L. Böhm, B. J. Walker, K. P. Musselman, N. C. Greenham, R. H. Friend, and A. Rao, Nat. Mater. 13, 1033 (2014).

16 J. J. Burdett, G. B. Piland, and C. J. Bardeen, Chem. Phys. Lett. 585, 1 (2013).

17 G. B. Piland, J. J. Burdett, D. Kurunthu, and C. J. Bardeen, J. Phys. Chem. C 117, 1224 (2013).

18 D. N. Congreve, J. Lee, N. J. Thompson, E. Hontz, S. R. Yost, P. D. Reusswig, M. E. Bahlke, S. Reineke, T. Van Voorhis, and M. A. Baldo, Science 340, 334 (2013).

19 L. Yang, M. Tabachnyk, S. L. Bayliss, M. L. Bohm, K. Broch, N. C. Greenham, R. H. Friend, and B. Ehrler, Nano Lett. 15, 354 (2014).

${ }^{20}$ R. E. Merrifield, Pure Appl. Chem. 27, 481 (1971).

21 J. J. Burdett and C. J. Bardeen, J. Am. Chem. Soc. 134, 8597 (2012).

${ }^{22}$ X. Wei, B. C. Hess, Z. V. Vardeny, and F. Wudl, Phys. Rev. Lett. 68, 666 (1992).

23 L. S. Swanson, J. Shinar, and K. Yoshino, Phys. Rev. Lett. 65, 1140 (1990).

24 L. Yarmus, J. Rosenthal, and M. Chopp, Chem. Phys. Lett. 16, 477 (1972).

25 S. L. Bayliss, K. J. Thorley, J. E. Anthony, H. Bouchiat, N. C. Greenham, and A. D. Chepelianskii, Phys. Rev. B 92, 115432 (2015).
26 S. L. Bayliss, A. D. Chepelianskii, A. Sepe, B. J. Walker, B. Ehrler, M. J. Bruzek, J. E. Anthony, and N. C. Greenham, Phys. Rev. Lett. 112, 238701 (2014).

27 M. Wakasa, M. Kaise, T. Yago, R. Katoh, Y. Wakikawa, and T. Ikoma, J Phys. Chem. C 119, 25840 (2015).

28 J. Mezyk, R. Tubino, A. Monguzzi, A. Mech, and F. Meinardi, Phys. Rev. Lett. 102, 087404 (2009).

29 R. Liu, Y. Zhang, Y. Lei, P. Chen, and Z. Xiong, J. Appl. Phys. 105, 093719 (2009).

30 T. N. Singh-Rachford and F. N. Castellano, Coord. Chem. Rev. 254, 2560 (2010).

31 D. Y. Kondakov, Phil. Trans. R. Soc. A 373, 20140321 (2015).

32 Throughout this article, we take an axially symmetric triplet exciton and neglect the non-axial contribution to $\hat{H}_{\mathrm{zfs}}, E\left(\hat{S}_{i, x}^{2}-\hat{S}_{i, y}^{2}\right)$.

33 For weakly interacting triplets with a zero-field splitting parameter $E=0$, there are two states with singlet character at both zero and high magnetic field and hence the PL in Fig. 1(b) saturates at its zero field value.

34 R. P. Groff, R. E. Merrifield, P. Avakian, and Y. Tomkiewicz, Phys. Rev. Lett. 25, 105 (1970).

35 H. Sternlicht and H. M. McConnell, J. Chem. Phys. 35, 1793 (1961).

36 S. T. Roberts, R. E. McAnally, J. N. Mastron, D. H. Webber, M. T. Whited, R. L. Brutchey, M. E. Thompson, and S. E. Bradforth, J. Am. Chem. Soc. 134, 6388 (2012).

37 A. Köhler and D. Beljonne, Adv. Func. Mat. 14, 11 (2004).

38 A. P. Monkman, H. D. Burrows, L. J. Hartwell, L. E. Horsburgh, I. Hamblett, and S. Navaratnam, Phys. Rev. Lett. 86, 1358 (2001).

39 L. R. Weiss, S. L. Bayliss, F. Kraffert, K. J. Thorley, J. E. Anthony, R. Bittl, R. H. Friend, A. Rao, N. C. Greenham, and J. Behrends, (unpublished).

40 E. A. Weiss, M. A. Ratner, and M. R. Wasielewski, J. Phys. Chem. A 107, 3639 (2003).

41 M. Chabr, U. Wild, J. Fünfschilling, and I. ZschokkeGränacher, Chemical Physics 57, 425 (1981).

42 R. Wang, C. Zhang, B. Zhang, Y. Liu, X. Wang, and M. Xiao, Nat. Commun. 6 (2015).

43 Analogous to $\gamma_{-}$in Eq. 1.

44 A. M. Müller, Y. S. Avlasevich, W. W. Schoeller, K. Müllen, and C. J. Bardeen, J. Am. Chem. Soc. 129, 14240 (2007).

45 J. Zirzlmeier, D. Lehnherr, P. B. Coto, E. T. Chernick, R. Casillas, B. S. Basel, M. Thoss, R. R. Tykwinski, and D. M. Guldi, Proc. Natl. Acad. Sci. 112, 5325 (2015).

46 N. V. Korovina, S. Das, Z. Nett, X. Feng, J. Joy, R. Haiges, A. I. Krylov, S. E. Bradforth, and M. E. Thompson, J. Am. Chem. Soc. 138, 617 (2016).

47 S. Lukman, A. J. Musser, K. Chen, S. Athanasopoulos, C. K. Yong, Z. Zeng, Q. Ye, C. Chi, J. M. Hodgkiss, J. Wu, et al., Adv. Func. Mat. 25, 5452 (2015). 